\title{
EVALUATION OF BULLET TRAIN VIBRATION FOR RESIDENTS NEAR RAILWAYS
}

\author{
Yoshiharu YONEKAWA \\ National Institute of Industrial Health, Nagao 6-chome, \\ Tama-ku, Kawasaki 213 Japan
}

(Received April 25, 1977)

\begin{abstract}
Sensation of an intermittent vibration such as bullet train vibrations was equalized to the sensation of a continuous random vibration which possessed frequency characteristics of $6 \mathrm{~dB} /$ oct between 8 and $80 \mathrm{~Hz}$. The values of the intermittent vibration read by the vibration level meter standardized in this country (physical quantity) corresponded to the equal sensation value of the random vibration (sensation quantity). This type of random vibration was able to use as the model of the bullet train vibrations. It was noticed in the experiment of sensation comparison that the longer the length of rest time between the sample train vibration and the compared random vibration was, the lower the equal sensation values became.

Then, emotion caused by this continuous model random vibration was rated in an evaluation scale in 5 steps (scarcely unpleasant, slightly unpleasant and so on). The experiment was carried out on a vibration table and on 10 male subjects. The first step "scarcely unpleasant" corresponded to $65 \mathrm{dBVL}$ (vibration level, rms acceleration levels weighted by frequency characteristic simulated for human vibration response, in $\mathrm{dB}$ ) the second step "slightly unpleasant" to $80 \mathrm{dBVL}$ and the third step "unpleasant" to $90 \mathrm{dBVL}$ for vertical vibration. For horizontal vibration, the third step corresponded to $92 \mathrm{dBVL}$. Thus, the emotional response could be estimated from the observed values obtained from the bullet trains.
\end{abstract}

Evaluation methods of vibrations with reference to the industrial health have lately been studied in the fleld of physiology and behaviorogy. The methods for evaluation on continuous sinusoidal vibrations have been standardized internationally in ISO-2631' ${ }^{1}$. Intermittent vibrations such as bullet train vibrations have recently been searched from the view point of public nuisance. These papers concerning the bullet train vibrations reported mainly measurements of the vibrations in various conditions on elevated trucks or in cuttings in relation to vibration attenuation at some distance from railways ${ }^{2)}$. The others were questionnaires of emotional response in residents near the rail roads ${ }^{3}$. Few papers reported the evalation methods for the bullet train vibration using a vibration table.

Thus, sensation of the bullet train vibrations was converted to that of usual continuous vibrations such as a sinusoidal vibration or a continuous random vibration. A 


\section{Y. YONEKAWA}

main factor to affect the equal sensation values, such as a duration of rest time inserted between the train vibration and the reference continuous vibration, was also checked at that time. After conversion of sensation of the intermittent vibrations to that of the continuous vibrations, the emotion responses like "unpleasant" were examined using the continuous vibration with equalized values known. Based on the results of these experiments, we intended to establish the evaluation method of the bullet train vibrations to serve as an aid to find a solution of public nuisance problem.

\section{Experimental Procedures}

The whole experiments were carried out on 10 normal male subjects who were the ages from 27 to 50 years old and did not get accustomed to vibrations.

\section{Equal sentation observation}

Vibrations caused by the bullet train on the new Tokaido trunk line (Shinkansen) were presented as sample vibrations for our psychological experiment. They were reproduced on a vibration table of electro-dynamic type on which subjects were asked to sit and to compare sensation of the train vibration with that of reference vibrations according to psycho-acoustical technique. The reference vibration was adjusted by the subject until he obtained equal sensation. The reference vibration was sinusoidal at $20 \mathrm{~Hz}$, because $20 \mathrm{~Hz}$ was predominant in lower frequency parts on spectra of the bullet train vibrations. As a reference vibration, a random vibration which was generated by a zenor diode used as a voltage regulator and passed through a weighting circuit with frequency characteristic of $6 \mathrm{~dB} /$ oct between 8 and $80 \mathrm{~Hz}$ was used. This weighting circuit was inserted for the purpose that the subject was facilitated on subjective judgement of just equal sensation because this random vibration could produce similar sensation of the sample vibration.

The bullet train vibrations were observed on ground at a rectangular distance of $10 \mathrm{~m}$ from the center of railways of Shinkansen whether in the cutting or on the elevated trucks. They were measured with a vibration meter (Rion Co.) in vertical and horizontal directions simultaneously and their vibration accelerations were recorded with a data recorder. The typical parts of vibrations were recorded again on an endless tape and reproduced on the vibration table through an attenuator, an equalizer of analogue type and a power amplifier. The equalizer was used to compensate the frequency characterisic of the vibration table of electro-dynamic type to be flat.

The subject was given a sample vibration only once for about $10 \mathrm{sec}$ and forced to memorize the extent of its perception. After a $5 \mathrm{sec}$ rest, the reference vibration was offered and continued until the subject just reached the equal sensation. When the equal sensation was detected, the level of the reference vibration on the vibration table was measured by the vibration meter set on the table to determine the equal seneation value. During this sensation equalization, the level of the reference vibration could be 


\section{VIBRATION EVALUATION FOR RESIDENTS NEAR RAILWAYS}

adjusted by the subject with a variable resister inserted between the generator of an electric oscillator of the reference vibration and the power amplifier. The subject controlled vibration magnitude according to the adjustment method by which the vibration level was started to change in ascending series. Whereas a tester switched the sample vibration to the reference vibration. The subjects were randomly given each set of combinations of vibrations which consisted of two vibrations (in the cutting and on the elevated truck) and three vibration levels to avoid a sequential error. The subjects were given the sample vibrations of three levels of 75,85 and $95 \mathrm{dBVL}$ which had been observed actually or somewhat larger than the real vibration level of the bullet train. The value dBVLmeans the weighted vibration acceleration level as $20 \log a / a_{\text {ref }}$ where a is rms vibration acceleration value $\mathrm{m} / \mathrm{sec}^{2}$ and $a_{\mathrm{ref}}$ is $10^{-5} \mathrm{~m} / \mathrm{sec}^{2}$. This weighting circuit possesses the frequency characteristic simulated with the human vibration response in both vertical and horizontal directions standardized internationally in ISO-2631. The definition of vibration level in $\mathrm{dBVL}$ or VL value and the specification of the vibration levelmeter have been standardized by Ministry of Trade and Industry in Japan, The level of the sinusoidal vibration at $20 \mathrm{~Hz}$ was also measured with the value of dBVL. The vibration acceleration value without passing this weighting circuit was also used as $\mathrm{dBAL}$ in this paper.

\section{Effect of duration of rest time}

It has been believed that the duration of rest time between the sample and the reference vibrations affected the equal sensation value. To investigate this effect, the duration was changed in 4 grades of $5,30,60$, and $90 \mathrm{sec}$. In this examination, the vibration caused by the train only in the cutting was selected as the sample vibration and the reference vibration was the random vibration mentioned above. The subjects were given the sample vibrations at the levels of 80 and $90 \mathrm{dBVL}$. During the rest time, the subjects were offered a back ground vibration of $10 \mathrm{~dB}$ less level than the sample vibration so that they might rightly judge the equal sensaton. The same random vibration mentioned above was used as the back ground vibration.

\section{Rating scale on the evaluation of random vibration sensation}

Two types of the bullet train vibrations could be equalized by subjective judgements to one type of the random vibration. Namely, the intermittent vibration could be converted by subjective rating to the continuous vibrations. The random vibration of the one type with the levels os $65,75,85$ and $95 \mathrm{dBVL}$ were subjectively rated into 5 steps

Table 1. Evaluation scales for vibration sensation.

\begin{tabular}{lllll}
\hline \multicolumn{1}{c}{1} & 2 & \multicolumn{2}{c}{3} & \multicolumn{1}{c}{4} \\
\hline Scarcely unpleasant & Slightly unpleasant & Unpleasant & Very unpleasant & Extremely unpleasant \\
Scarcely nervous & Slightly nervous & Nervous & Very nervous & Extremely nervous \\
Scarcely annoying & Slightly annoying & Annoying & Very annoying & Extremely annoying \\
\hline
\end{tabular}




\section{Y. YONEKAWA}

of a psycholgical evaluation scale instead of treating many types of actual bullet train vibrations. The random vibrations with the continuous duration of $10 \mathrm{sec}, 1 \mathrm{~min}$ and 5 min were examined to check the effect of their exposure time on the emotional response. Each set of combinations consisting of three exposure times and four vibration levels was randomly given to the subject. Three types of words with a semantic difference were used for analysis of feeling such as "unpleasant" "nervous" and "annoying". Their evaluation scales were as follows, for example in "unpleasant" scale, 1. scarcely unpleasant, 2. slightly unpleasant, 3. unpleasant, 4. very unpleasant,and 5. extremely unpleasant as shown in Table 1.
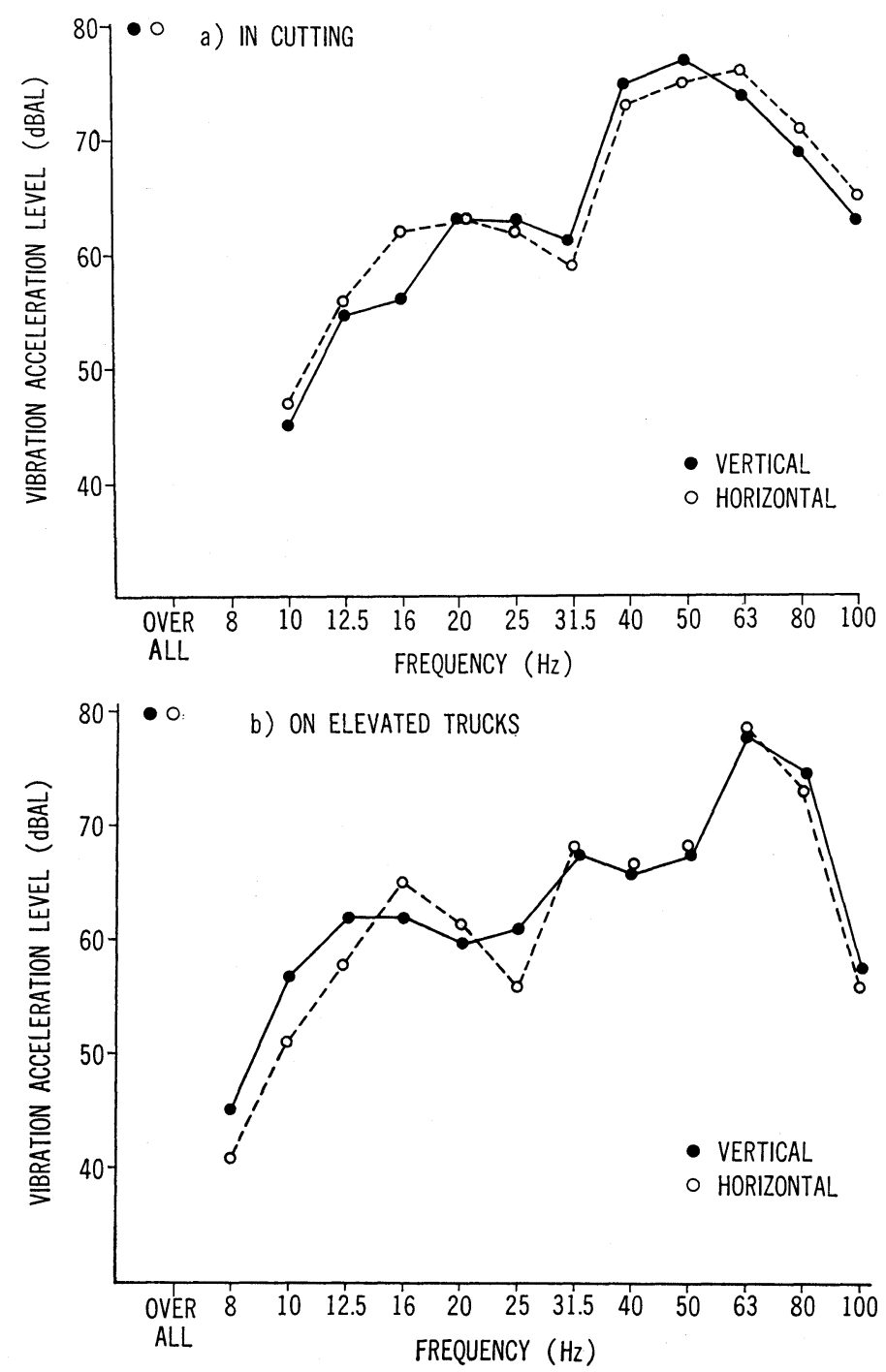

Fig. 1. Frequency spectra of vibration acceleration of bullet trains of Tokaiodo Shinkansen line observed on the ground at vertical distance of $10 \mathrm{~m}$ from the central point of the railways. (a) railways the cutting and (b) that on the elevated trucks. 


\section{VIBRATION EVALUATION FOR RESIDENTS NEAR RAILWAYS}

\section{Results AND Discussion}

\section{Frequency spectra of sample vibrations}

Typical frequency spectra of acceleration of sample vibrations are shown in Fig. 1, $\mathrm{a}$ and $\mathrm{b}$. The overall level was adjusted at $80 \mathrm{dBAL}$ for convenience. The vibration spectra in the cutting and on the elevated trucks differ with each other. There are two peaks and one notch in the spectra of both vibrations. The lower and upper frequency components $(16$ and $63 \mathrm{~Hz}$ ) on the elevated trucks (b) are more predominant than those $(20$ and $60 \mathrm{~Hz}$ ) in the cutting (a). The spectral difference of vibrations in both vertical and horizontal directions can not be seen on these two kinds of vibrations.

\section{Equal sensations sinusoidal vibration at $20 \mathrm{~Hz}$}

The sample vibrations were equalized in sensation to the sinusoidal vibration at 20 Hz. As shown in Fig. 2, $a$ and $b$, the differences between the equal sensation value observed in $\mathrm{dBVL}$ and the $\mathrm{VL}$ value of the sample vibration are plotted against the vibration level of the sample vibrations. In the vertical vibration (Fig. 2, a) at $85 \mathrm{dBVL}$ the subjects equate the sample vibration sensation to almost the same VL value as that of the sinusoidal vibration at $20 \mathrm{~Hz}$. On the contrary, at $75 \mathrm{dBVL}$ of the sample vibration, they judge the equal sensation by about $5 \mathrm{~dB}$ larger than the VL value of sample vib-

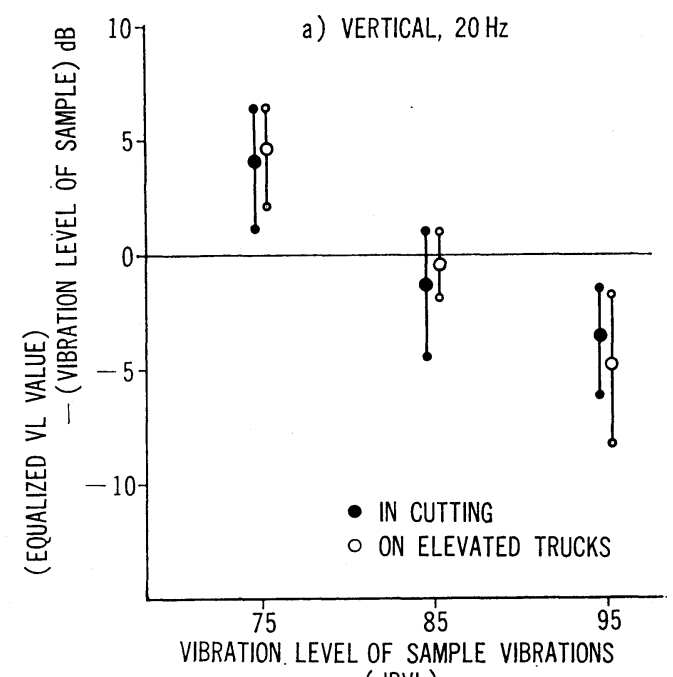

(dBVL)

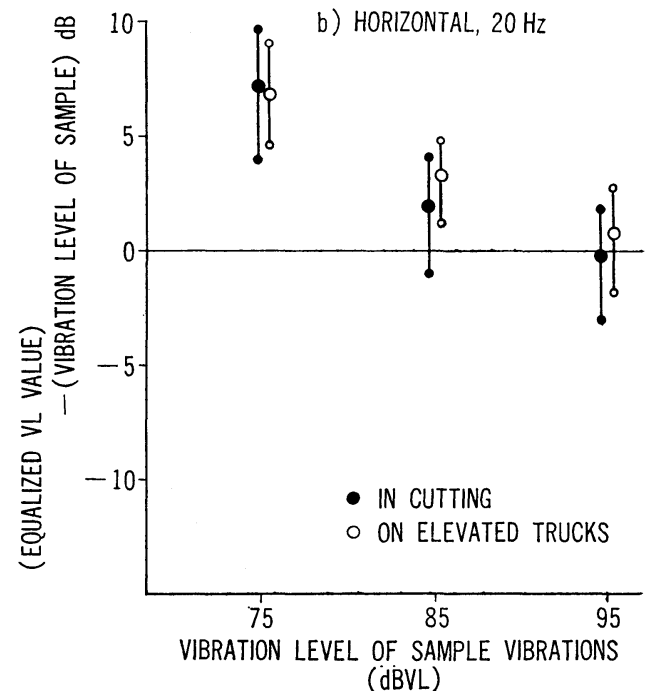

(dBVL)

Fig. 2. Equal sensation values of sinuoidal vibration at $20 \mathrm{~Hz}$ compared with the bullet train vibrations. a) vibrations in vertical direction and b) those in horizontal direction. The ordinate shows the difference between VL value of the sinusoidal vibration 20 $\mathrm{Hz}$ just equalized in sensation and VL value of the sample vibration. The abscissa is VL values of the sample vibrations. Averages and standard deviations are shown in the figures on 10 male subjects. 


\section{Y. YONEKAWA}

ration. And at $95 \mathrm{dBVL}$, the equal sensation is by about $5 \mathrm{~dB}$ less than the sample vibration.

In the horizontal vibration, the equal sensation value is in accord with VL value of the sample vibration at its $95 \mathrm{dBVL}$. When the level of sample vibration lowers, the difference between the equal sensation value and the VL value of the sample vibration becomes larger. It may be assumed that if the horizontal sample vibration above 95 dBVL was given the equal sensation valne might be lowered. The trend of which the sample vibration with higher level is equalized in sensation to the reference vibration with lower level and vice versa, may be partially explained by the central tendency of sensation judgement in psychology. The equal sensation values of the sample vibrations do not differ significantly in a statistic test between those obtained in the cutting and on the elevated trucks. The difference between the equal sensation value at $20 \mathrm{~Hz}$ and the VL value of the sample vibration has clearly linear tendency against the level of sample vibration. The sinusoidal vibration at $20 \mathrm{~Hz}$. therefore, can not be suitable for equalization in sensation to the train vibration.

\section{Equal sensation of random vibration}

In order to search the reasonable reference vibration in the equal sensation experiment, the sample vibration was compared with the random vibration mentioned already. The results are shown in Fig. 3, a and b. The effect of the sample vibration level on

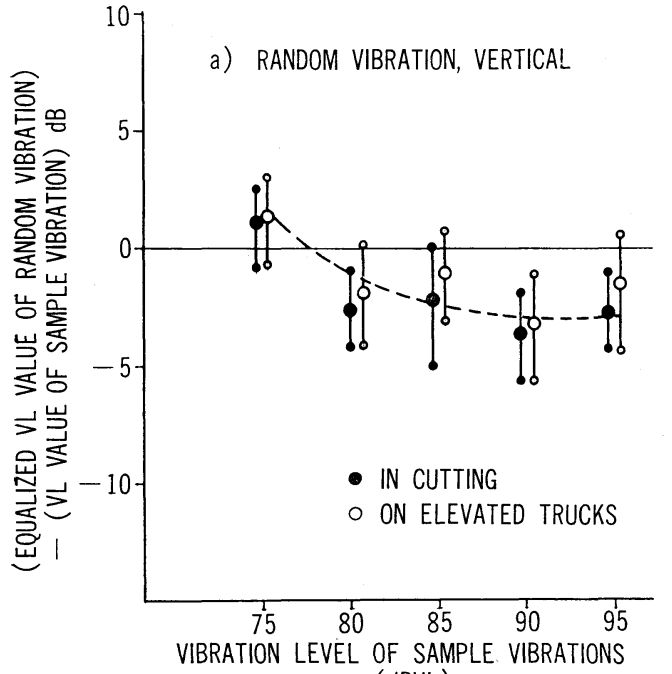
$(\mathrm{dBVL})$

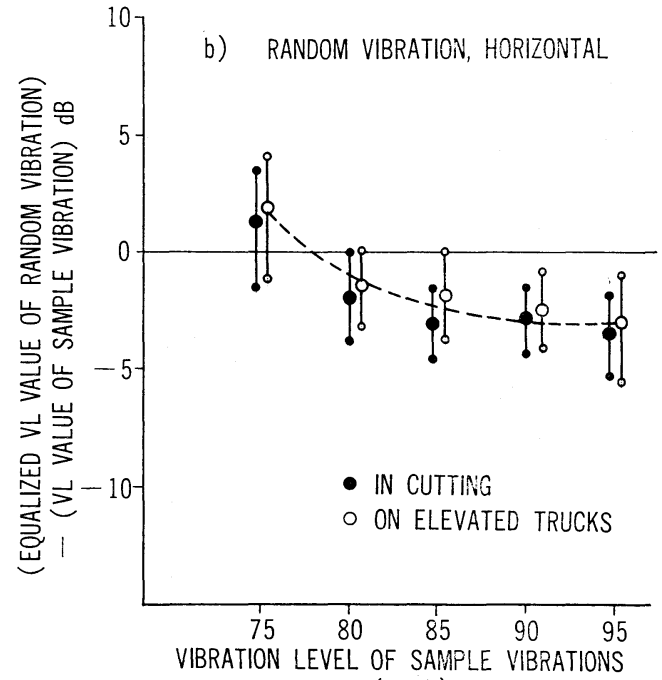

( $d B V L)$

Fig. 3. Equal sensation values of random vibrations compared with the bullet train vibrations. a) vibrations in vertical direction and $b$ ) those in horizontal direction. The ordinate shows the difference between VL value of the random vibratons just equalized in sensation and VL value of the sample vibration. The abscissa is VL values of the sample vibrations. Averages and standard deviations are shown in the figures on 10 male subjects. 


\section{VIBRATION EVALUATION FOR RESIDENTS NEAR RAILWAYS}

the equal sensation value of random vibration does not have the linear relation as compared with the case of the sinusoidal vibration at $20 \mathrm{~Hz}$ (Fig. 2, a and b) and the central tendency decreases in the random vibration. Besides, the equal sensation values of the random vibration is independant upon the kinds of vibration (in the cutting or on the elevated truck). Both the data for vertical and horizontal vibrations can be approximated with the same curve, on which the value of (equalized VL-sample's VL) indicate positive value below $78 \mathrm{dBVL}$ and negative value above $78 \mathrm{dBVL}$ and then saturate at the constant value. The standard deviations at each VL value on this curve are almost similar and fluctuate within about $\pm 2.5 \mathrm{~dB}$. The bias of this curve from the zero line (solid line in the figure) is not more than $\pm 3 \mathrm{~dB}$ ranging from 75 to $95 \mathrm{dBVL}$.

Thus, the sensation of two kinds of train vibration can be converted to that of one kind of simple continuous random vibration with nearly the same VL value, though the bullet train vibration is not so long in its duration. As the sample vibrations used here are typical among the bullet train vibrations, it can be said that all train vibration with the level from 75 to $95 \mathrm{dBVL}$ can be converted to the random vibration having about the same VL value of the sample vibration measured by the vibration level meter.

\section{Effect of rest time on equal sensation}

The effect of length of the rest time on the equal sensation value is indicated in Fig. 4, and b. The longer the rest time, the lower the equal sensation value of the random vibration becomes. In additon to this lowering the level of equal sensation, the

a) EFFECT OF REST TIME, VERTICAL

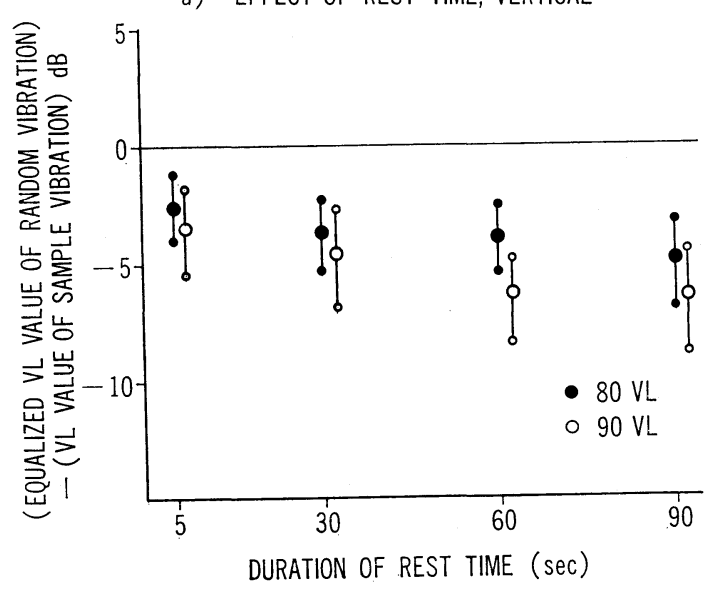

b) EFFECT OF REST TIME, HORIZONTAL

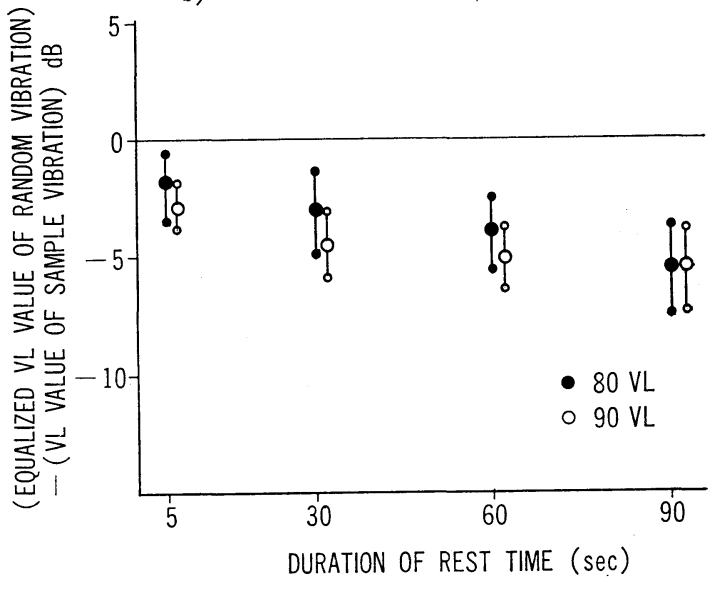

Fig. 4. Effect of duration of rest time inserted between the sample and the comparing vibrations on equal sensation values of random vibration. a) vibrations in vertical direction and b) those in horizontal direction. The ordinate shows the difference between VL value of the random vibrations just equalized in sensation and VL value of the sample vibration. The abscissa is duration of rest time (sec). Averages and standard deviations are shown in the figures on 10 male subjects. 


\section{Y. YONEKAWA}

standard deviation becomes somewhat larger, because memory of magnitude of the sample vibration turns to be vague for elapsing time. The change of equal sensation owing to the length of rest time is known as the time error in psychology, which could be found out not only in the loudness of sound, but also in the sensation of weight. On the date obtained from lapse of time, it is understood that the subject can hardly reproduce his memory with sufficient reliability. The present experiment, therefore, was carried out on the rest time of $5 \mathrm{sec}$ to facilitate the equal sensation judgement.

Curves of the difference between the equalized VL value and the VL value of the sample vibration are almost similar in both vertical and horizontal vibration. At $90 \mathrm{sec}$, $-6 \mathrm{~dB}$ was obtained as compared with the zero point (solid line in the figure). A little difference seems to be observed between the two data on the sample vibrations with the levels at 80 and $90 \mathrm{dBVL}$.

\section{Emotional response on evaluation scale}

The results of rating of sensation of the random vibration mentioned above on the evaluation scale are shown in Fig. 5. a, b and $c$ on average values obtained from 10 subjects. Their standard deviations are indicated in Table 2 . The duration of the random vibrations given to the subject were changed $10 \mathrm{sec}, 1 \mathrm{~min}$ and $5 \mathrm{~min}$ on the vertical vibration and the vibration level was varied from 65 to $95 \mathrm{dBVL}$. The dotted curves are plotted similarly on the three different semantic words to check the trend of the data observed. There are no clear difference among criteria of "unpleasant", "nervous" and "annoying", but rather similar tendency on the exposure time of the random vibration. These results, therefore, denote that the subject may judge the vibration perception in

Table 2. Averages and thiseir standard deviation on evaluation scale.

\begin{tabular}{|c|c|c|c|c|c|}
\hline & \multicolumn{4}{|c|}{ Vertical } & \multirow{2}{*}{$\frac{\text { Horizontal }}{1 \mathrm{~min}}$} \\
\hline & $\begin{array}{l}\text { Vibration Level } \\
\quad(\mathrm{dBVL})\end{array}$ & $10 \mathrm{sec}$ & $1 \mathrm{~min}$ & $5 \mathrm{~min}$ & \\
\hline \multirow[t]{4}{*}{ Unpleasant } & 65 & 1.0 & 1.0 & 1.0 & 1.0 \\
\hline & 75 & $1.6 \pm 0.70$ & $1.4 \pm 0.52$ & $1.7 \pm 0.67$ & $1.1 \pm 0.32$ \\
\hline & 85 & $2.1 \pm 0.74$ & $2.3 \pm 0.95$ & $2.8 \pm 0.92$ & $1.6 \pm 0.70$ \\
\hline & 95 & $3.2 \pm 1.03$ & $4.3 \pm 0.67$ & $4.0 \pm 0.82$ & $3.3 \pm 1.06$ \\
\hline \multirow[t]{4}{*}{ Nervous } & 65 & $1.4 \pm 0.52$ & $1.4 \pm 0.52$ & $1.4 \pm 0.52$ & $1.3 \pm 0.48$ \\
\hline & 75 & $1.7 \pm 0.63$ & $1.6 \pm 0.56$ & $2.1 \pm 0.52$ & $1.7 \pm 0.48$ \\
\hline & 85 & $2.3 \pm 0.63$ & $2.4 \pm 0.74$ & $2.5 \pm 0.57$ & $2.3 \pm 0.48$ \\
\hline & 95 & $3.5 \pm 0.82$ & $3.8 \pm 0.82$ & $3.8 \pm 0.82$ & $3.9 \pm 0.99$ \\
\hline \multirow[t]{4}{*}{ Annoying } & 65 & $1.2 \pm 0.42$ & 1.0 & $1.3 \pm 0.48$ & 1.0 \\
\hline & 75 & $2.0 \pm 0.67$ & $1.8 \pm 0.63$ & $2.0 \pm 0.67$ & $1.4 \pm 0.52$ \\
\hline & 85 & $2.4 \pm 0.97$ & $2.6 \pm 0.70$ & $3.1 \pm 0.57$ & $2.1 \pm 0.74$ \\
\hline & 95 & $3.8 \pm 0.63$ & $4.3 \pm 0.67$ & $4.2 \pm 0.79$ & $3.6 \pm 0.97$ \\
\hline
\end{tabular}




\section{VIBRATION EVALUATION FOR RESIDENTS NEAR RAILWAYS}
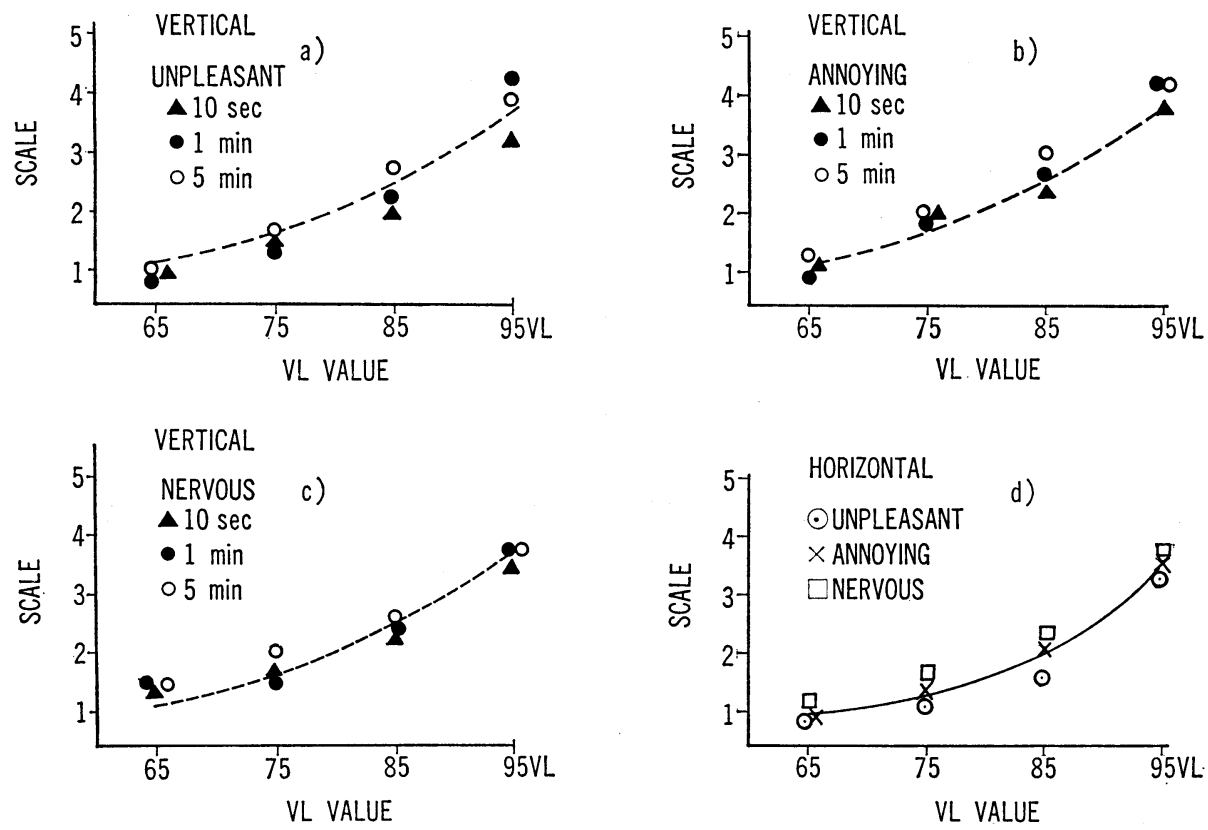

Fig. 5. Emotional response of random vibration on evaluation scale in 5 steps. The ordinate shows the scale such as 1 . scarcely unpleasant, 2 . slightly unpleasant, 3 . unpleasant, 4. very unpleasant and 5. extremely unpleasant. The other semantic different words are "nervous" and "annoying". The abscissa indicates the VL value of random vibration from 65 to $95 \mathrm{VL}$ with duration of $10 \mathrm{sec}, 1 \mathrm{~min}$ and $5 \mathrm{~min}$. (a), (b), (c) emotional responses of vertical vibration on the different impressing time of vibration concerning three different words, and (d) those of horizontal vibration concerning three different words. The data observed at each point indicate averages on 10 male subjcts.

the early stage after giving of the vibration. The results on the horizontal random vibration for $1 \mathrm{~min}$ duration are shown in Fig. 5 , d. The tendency is in some degree different from that of the vertical vibration, because the different weighting circuits were used for measuring the vertical and the horizontal vibrations. That is, the VL value was read out by the vibration level meter of which frequency characteristics were separately weighted with each other by the human vibration sensation for the vertical and the horizontal vibrations. It is thought that since the weighted frequency characteristic are not sufficiently in accordance with the human sensation, a little difference mentioned above may be observed.

In these figures, the first stage on the scale corresponds to about $65 \mathrm{dBVL}$, the second stage to $80 \mathrm{dBVL}$ and the third stage to $90 \mathrm{dBVL}$ for vertical vibrations without regard to three semantic different words. For horizontal vibrations, the first stage corresponds to $65 \mathrm{dBVL}$, the second stage to $85 \mathrm{dBVL}$ and the third stage to $92 \mathrm{dBVL}$.

It is supposed that these results can not be applied directly to the problem of public nuisance, because the results obtained on the vibration table are somewhat diffierent from the data observed by residents who live in the house, suffer from the vibration and are 


\section{Y. YONEKAWA}

very nervous to the vibration. The difference between the laboratory experiment and the field survey for public nuisance comes from the quality of the subjects and the environmental condition for the observation. It, however, is believed that under consideration of this difference, the values to subtract about $10 \mathrm{~dB}$ from the observed values in the results (Fig. 5) can be used for evaluation of the vibration to the public nuisance, for example, the third stage, in this case, decreases to $80 \mathrm{dBVL}$ for the vertical vibration. This $10 \mathrm{~dB}$ difference comes from the vibration regulation law in Environmental Agency)

\section{Acknowledgment}

The auther is indebted to Dr. H. Sakabe, Director of this instiute, Dr. S. Koike, Professor of Showa Medical School and Dr. T. Miwa, Senior member of this institute for their valuable suggestions.

\section{REFERENCES}

1) ISO-2631-1974 (E)

2) Kobayashi Institute of Physical Research (1973), Shindo Kogai ni Kansuru Chosa Kenkyu Hokokusho (Reports of Investigations on Vibration Pollution), Tokyo. (in Japanese)

3) Nishinomiya, G. (1976). J. Acoust. Soc. Jap., 32, 147 (in Japanese)

4) Environment Agency (1976) Vibration Regulation Law. 